Chirurg 2015 $\cdot 86: 496$

DOI 10.1007/s00104-015-3020-2

Online publiziert: 30. April 2015

(c) Springer-Verlag Berlin Heidelberg 2015
O. Strobel · M.W. Büchler

Klinik für Allgemein-, Viszeral- und Transplantationschirurgie, Universität Heidelberg

\section{Erhöhte Mortalität durch mangelnde Erfahrung mit der minimal-invasiven Pankreatoduodenektomie}

\section{Hintergrund und Fragestellung}

Die Pankreatoduodenektomie (PD) gehört zu den anspruchsvollsten viszeralchirurgischen Eingriffen und hat eine hohe Komplikationsrate. Trotzdem werden insbesondere in den USA auch für die PD minimal-invasive Techniken propagiert. Als Argumente für die minimalinvasive PD (miPD) werden ein geringeres operatives Trauma, eine kürzere Liegedauer und eine raschere Erholung proklamiert. Nachteile werden insbesondere in einer Erschwerung der ohnehin schon anspruchsvollen Rekonstruktion im Vergleich zur offenen PD (oPD) gesehen. Nachdem von einzelnen Zentren gute Ergebnisse der miPD gezeigt werden konnten, findet die miPD in den USA auch aus Marketinggründen eine zunehmende Verbreitung.

Adam et al. haben die Anwendungsmuster und die Kurzzeitergebnisse der miPD im Vergleich zur oPD in einer repräsentativen Querschnittsstudie untersucht.

\section{Methoden}

Aus der National Cancer Database, die Behandlungsergebnisse von mehr als 1500 akkreditierten Zentren und damit von ca. $75 \%$ der neu diagnostizierten Krebsfälle in den USA umfasst, wurden Patienten iden- tifiziert, bei denen in den Jahren 2010 und 2011 eine PD durchgeführt wurde. Anhand deskriptiver Statistik und multivariater Analysen wurden die Anwendungsmuster und perioperativen Ergebnisse der miPD (laparoskopisch und robotisch) und oPD verglichen. Konvertierte PD wurden in der miPD-Gruppe analysiert (,intention-to-treat").

zahl. Die Ergebnisse hatten Bestand nach Ausschluss der konvertierten Fälle.

\section{Diskussion}

Die Autoren folgern, dass die miPD mit einer erhöhten 30-Tage-Mortalität assoziiert ist und raten zur Vorsicht bezüglich der Ausbreitung dieses Verfahrens.

Die guten Ergebnisse der miPD in

\section{Ergebnisse}

Bei insgesamt 7061 Patienten wurden 983 (14\%) miPD und 6078 (86\%) oPD durchgeführt. Die Konversionsrate betrug 30\% $(\mathrm{n}=294)$. In 246 Kliniken wurden innerhalb der 2 Jahre zwischen einer und 81 miPD durchgeführt, wobei $92 \%$ der Kliniken $\leq 10$ miPD und 50\% der Kliniken lediglich eine miPD durchführten. Patienten mit miPD hatten im Vergleich zu Patienten mit oPD häufiger Komorbiditäten ( $38 \%$ vs. $34 \%)$, wurden häufiger in akademischen Zentren operiert ( $71 \%$ vs. $63 \%)$ und hatten häufiger neuroendokrine Tumoren ( $11 \%$ vs. $8 \%$ ). $85 \%$ der miPD und $86 \%$ der oPD wurden bei Adenokarzinom durchgeführt. Die nicht adjustierte 30-Tage-Mortalitätsrate lag bei 4,8\% nach miPD und 3,7\% nach oPD. Die multivariate Analyse bei Patienten mit Adenokarzinom ergab nach Adjustierung keine Unterschiede zwischen miPD und oPD bezüglich des R-Status, der Anzahl analysierter Lymphknoten, der Liegedauer und der 30-Tage-Wiederaufnahmerate. Die adjustierte 30-Tage-Mortalität war nach miPD signifikant höher als nach oPD (Odds Ratio: 1,87; Konfidenzintervall: $1,25-2,80 ; \mathrm{p}=0,002)$. Dabei bestand für miPD und oPD ein klarer Trend für eine erhöhte Mortalität bei kleinerer Falldarauf spezialisierten Zentren sind keineswegs allgemeingültig: Während die miPD onkologisch adäquat durchführbar scheint (hinsichtlich R-Status und Lymphknotenzahl; Langzeitergebnisse stehen aus), ist sie bei Anwendung in der Breite nicht nur mit einer erhöhten Mortalität assoziiert, sondern verliert auch die postulierten Vorteile des minimal-invasiven Verfahrens (raschere Erholung, kürzere Liegedauer).

Die sichere Durchführung einer miPD macht eine hohe Erfahrung in minimal-invasiver und Pankreaschirurgie notwendig und hat eine lange Lernkurve. Die miPD sollte daher nur in entsprechenden Zentren durchgeführt werden und ihre Sinnhaftigkeit muss hier anhand der perioperativen und onkologischen Ergebnisse unter Adjustierung des bestehenden Selektionsbias kritisch reevaluiert werden.

\section{Korrespondenzadresse}

\section{PD Dr. O. Strobel}

Klinik für Allgemein-,

Viszeral- und Transplantationschirurgie, Universität Heidelberg

Im Neuenheimer Feld 110, 69120 Heidelberg

Oliver.Strobel@med.uni-heidelberg.de

Interessenkonflikt. O. Strobel und M.W. Büchler geben an, dass kein Interessenkonflikt besteht. 